ARTICLE

DOI: $10.1038 / \mathrm{s} 41467-017-00594-5$

\title{
interleukin-11 induces and maintains progenitors of different cell lineages during Xenopus tadpole tail regeneration
}

Hiroshi Tsujioka (1) ${ }^{1}$, Takekazu Kunieda (i) ${ }^{1}$, Yuki Katou ${ }^{2}$, Katsuhiko Shirahige ${ }^{2}$, Taro Fukazawa (D) ${ }^{1} \&$ Takeo Kubo $^{1}$

Unlike mammals, Xenopus laevis tadpoles possess high ability to regenerate their lost organs. In amphibians, the main source of regenerated tissues is lineage-restricted tissue stem cells, but the mechanisms underlying induction, maintenance and differentiation of these stem/progenitor cells in the regenerating organs are poorly understood. We previously reported that interleukin-11 (il-11) is highly expressed in the proliferating cells of regenerating Xenopus tadpole tails. Here, we show that il-11 knockdown (KD) shortens the regenerated tail length, and the phenotype is rescued by forced-il-11-expression in the KD tadpoles. Moreover, marker genes for undifferentiated notochord, muscle, and sensory neurons are downregulated in the KD tadpoles, and the forced-il-11-expression in intact tadpole tails induces expression of these marker genes. Our findings demonstrate that $i l-11$ is necessary for organ regeneration, and suggest that IL-11 plays a key role in the induction and maintenance of undifferentiated progenitors across cell lineages during Xenopus tail regeneration.

\footnotetext{
${ }^{1}$ Department of Biological Sciences, Graduate School of Science, The University of Tokyo, 7-3-1, Hongo, Bunkyo-ku, Tokyo 113-0033, Japan. ${ }^{2}$ Insutitute of Molecular and Cellular Biosciences, the University of Tokyo, 1-1-1, Yayoi, Bunkyo-ku, Tokyo 113-0032, Japan. Correspondence and requests for materials should be addressed to T.F. (email: tfukazawa@bs.s.u-tokyo.ac.jp)
} 
S ome lower vertebrates, such as fish and amphibians, have a prominent ability to regenerate their lost organs compared to mammals ${ }^{1}$. Xenopus laevis tadpoles can regenerate their lost tails, including all tissues that comprise tails, such as the notochord, muscle, spinal cord and other tissues, after amputation, and are used as model animals for the study of vertebrate organ regeneration. In the regeneration of $X$. laevis tails $^{2}$ or axolotl limbs ${ }^{3}$, the main sources of the regenerated organs are lineage-restricted tissue stem cells. Although the mechanisms underlying the synergistic induction, maintenance, and differentiation of these stem and/or progenitor blastema cells during organ regeneration are fundamental in organ regeneration, the detailed molecular mechanisms are not well understood. We previously reported that interleukin-11 (il-11) is highly expressed in the proliferating cells in regenerating $X$. laevis tadpole tails ${ }^{4}$, raising the possibility that IL-11 plays a crucial role in Xenopus tadpole tail regeneration.

IL-11 is a member of IL- 6 family, and its signalling cascade has been extensively studied in mammals ${ }^{5}$. IL-11 binds to both IL-11 receptor alpha (IL11RA) and IL-6 signal transducer (IL6ST, also known as GP130) ${ }^{6,7}$, and transduces signals through IL6ST $^{8}$. IL6ST is a receptor subunit common to all IL-6 family cytokines. Activated IL6ST phosphorylates signal transducer and activator of transcription (Stat) 1 and $3^{9}$, and phosphorylated Stat 1 and Stat3 translocate to the nucleus to activate the transcription of target genes ${ }^{10}$, 11 . IL-11 also activates the mitogen-activated protein/extracellular signal-regulated kinase kinase (MEK) pathway ${ }^{12}$, and the phosphatidylinositol-3 kinase (PI3K) pathway $^{13}$.

Some members of the IL-6 family are involved in regulating the differentiation of stem/progenitor cells. For example, IL-6 treatment differentiates B lymphocytes to antibody-forming cells $^{14}$. Leukaemia inhibitory factor (Lif) inhibits the differentiation of mouse embryonic stem cells ${ }^{15}, 16$. IL-11 treatment is reported to maintain the expression of undifferentiated markers in human embryonic stem cells ${ }^{17}$. $i l-11$ is also suggested to be involved in regeneration. il-11 is reported to be expressed in the regenerating heart of zebrafish, and forced expression of a dominant negative form of Stat3 inhibits the proliferation of cardiomyocytes and heart regeneration ${ }^{18}$. Based on these findings, Fang et al. speculated that IL-11 is a candidate upstream molecule of the Stat3 pathway that is responsible for the proliferation of cardiomyocytes during regeneration. The precise role of il-11 in regeneration of organs comprised of various tissues, however, is not clear.

Here, we produced $i l-11$ knockeddown (KD) tadpoles using the CRISPR/Cas9 system to show that il-11 is necessary for tail regeneration in $X$. laevis tadpoles. In addition, the shortened regenerated tails, a phenotype of the $i l-11 \mathrm{KD}$ tadpoles, is rescued by forced expression of $i l-11$ at the amputated tail stumps. In the amputated tail stumps of the $i l-11 \mathrm{KD}$ tadpoles, marker genes for undifferentiated notochord, muscle and sensory neurons are downregulated compared to control tadpoles. Furthermore, forced expression of il-11 in the intact tadpole tails induces expression of the markers for undifferentiated cells. Our results strongly suggest that IL-11 plays a key role in the induction and maintenance of undifferentiated progenitor cells across cell lineages during Xenopus tadpole tail regeneration.

\section{Results}

il-11 is induced after tail amputation. First, we examined the correlation between the cellular processes and $i l-11$ expression in regenerating Xenopus tadpole tails. Quantitative reverse transcription-polymerase chain reaction (qRT-PCR) of il-11 mRNA in the amputated tail stumps of tadpoles collected at 0 , $0.5,1,2$ and $5 \mathrm{~h}$ post amputation (hpa) showed that the il-11 expression began at 2 hpa (Fig. 1a), suggesting that $i l-11$ is related to early events immediately after tail amputation. We then examined $i l-11$ expression levels in later phases after amputation a

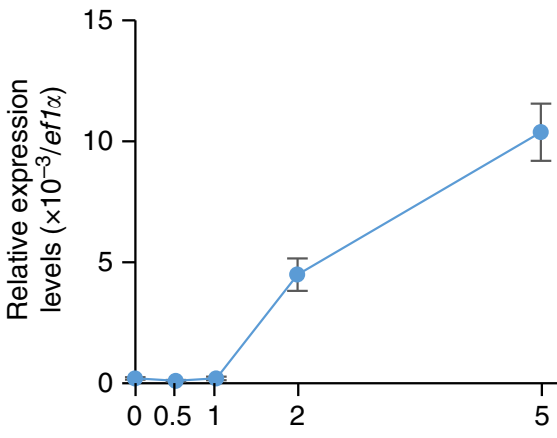

b

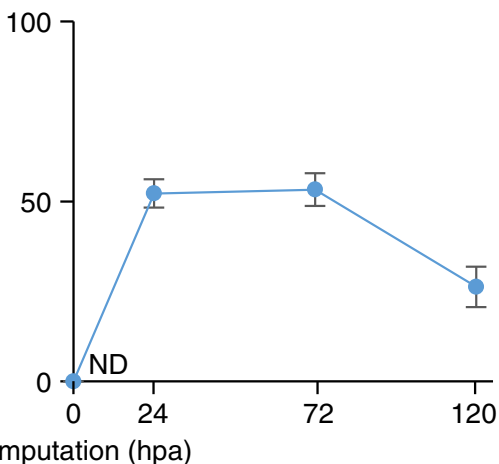

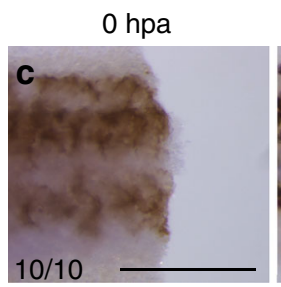
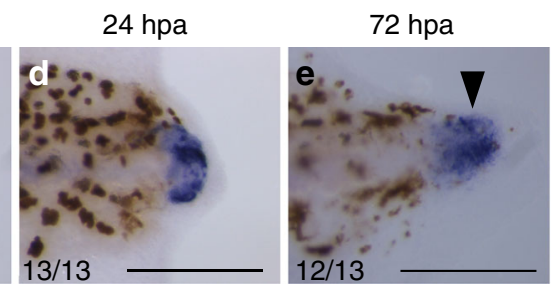

$120 \mathrm{hpa}$

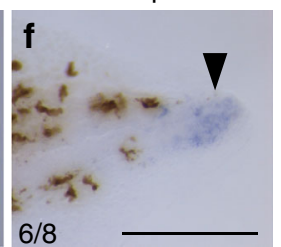

Fig. $1 \mathrm{il}-11$ is expressed at the tip of blastema during tail regeneration. $\mathbf{a}$, $\mathbf{b}$ Expression levels of il-11 were measured by qRT-PCR using RNA extracted from $\sim 20$ tadpoles. Tail stump tissues cut at the level of $0.5 \mathrm{~mm}$ anterior from the amputated plane were used. Vertical axes represent relative expression levels of $i l-11$ normalised by those of ef $1 \alpha$. Mean \pm s.e.m. $n=4$. ND: not detected. c-f WISH was performed using tadpoles fixed at $0 \mathbf{c}, 24 \mathbf{d}, 72 \mathbf{e}$, or 120 hpa $\mathbf{f}$. Anterior is to the left and dorsal is up. Blue/purple colour represents signals for il-11 expression. Brown pigments are melanophores of the tadpoles. Scale bars: $500 \mu \mathrm{m}$. Numbers at the bottom corner indicate the total ratio of tadpoles showing the corresponding expression pattern from at least two batches. Note that il-11 was expressed at the blastema tip at 72 and $120 \mathrm{hpa}$ (black arrowheads) 
a

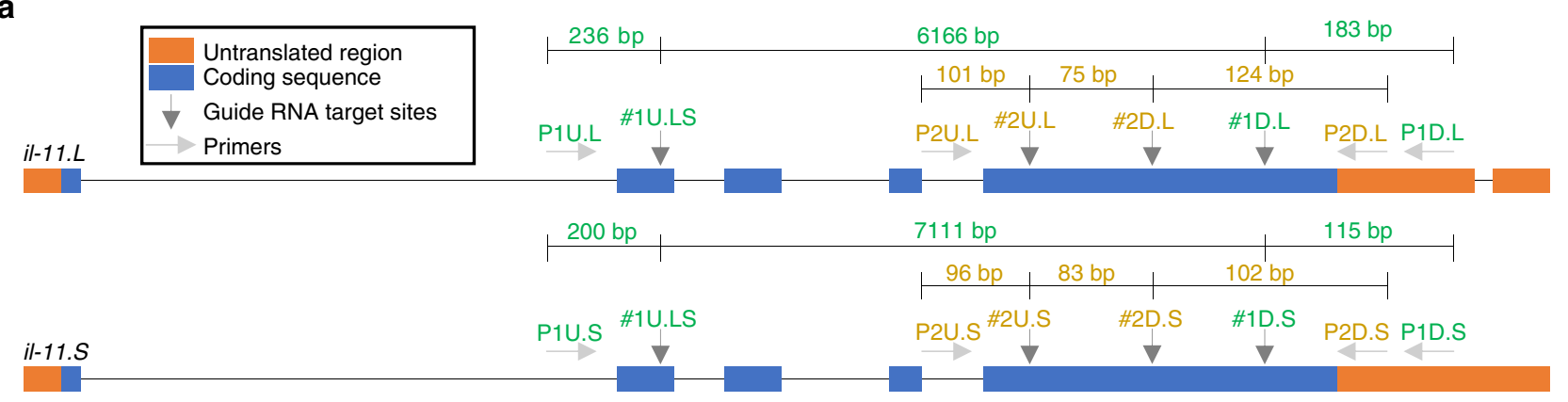

b

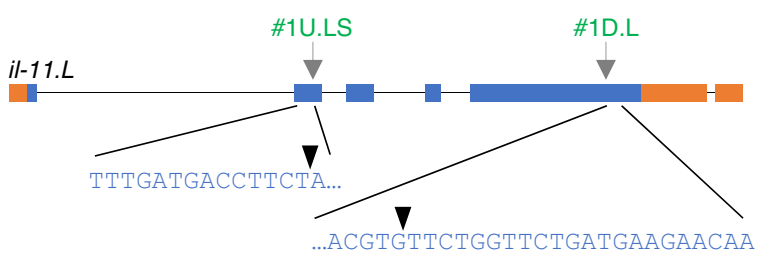

3/10 TTTGATGACCTTCT----------GGTTCTGAAGAAGAACAA

1/10 TTTGATGACCTTC--ACGTGTTCTGGTTCTGATGAAGAACAA

1/10 TTTGACCTTCA----------TCTGGTTCTGATGAAGAACAA

1/10 TTTGATGACCTTCT---------_GGTTTTGATGAAGAACAA

1/10 TTTGATGACCTTGG------------------------ACAA

1/10 TTTGATGACTTTCTGATGACATCTGGTTCTGATGAAGAACAA

1/10 TTTGATGACCTTCTTTCT--TTCTGGTTCTGATGAAGAACAA

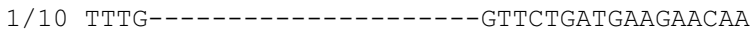

C

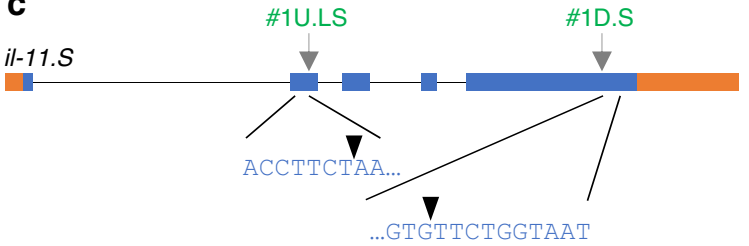

1/14 ACCTTCACGTAATTCTGGTAAT

1/14 ACCTTCT---------GGTAAT

1/14 ACCTTCGTCA--TTCTGGTAAT

2/14 ACCTTC---GTGTTCTGGTAAT

2/14 ACCTTCTA----TTCTGGTAAT

2/14 АССTTCT---------GGTAAT

1/14 ACCTTTT--------TGGTAAT

1/14 ACCT------------GGTAAT

1/14 ACCTTCTT----TTCTGGTAAT

1/14 ACCTTCT-----TTCTGGTAAT

1/14 AССТTCTAA----------AAT

e

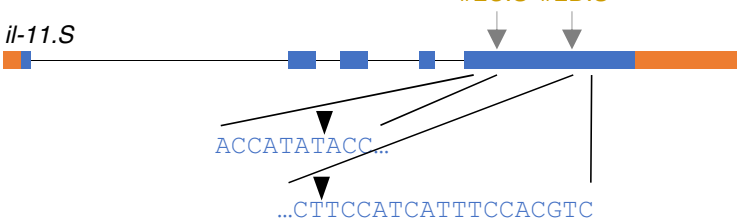

1/4 ACCAT-------CATTTCCACGTC

1/4 ACCATAT-CCATCATTTCCACGTC

1/4 ACCATATATGTCCATC----CGTC

1/4 ACCAT-TTCCATCATTTCCACGTC
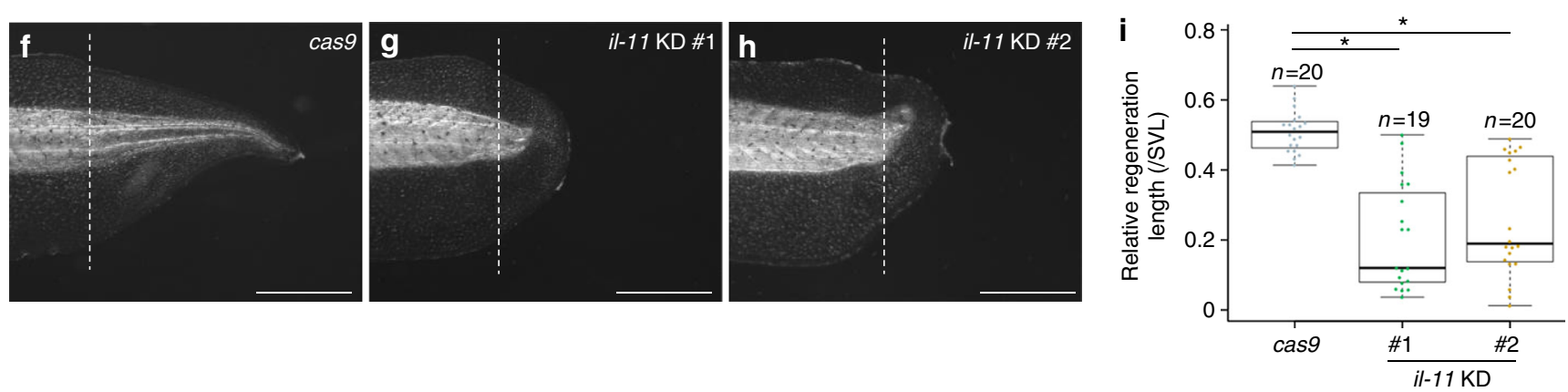

Fig. 2 Knockdown of il-11 in tadpoles. a Schematic drawing of genomic structure of il-11. Bars: introns, orange boxes: untranslated regions, blue boxes: coding sequences, dark grey vertical arrows: gRNA target sequences, light grey horizontal arrows: primers. b-e, Genomic sequences of tadpoles injected with cas 9 mRNA and gRNAs. Genomic DNA was extracted from all body of il-11 KD \#1 tailbud embryos $\mathbf{b}$, $\mathbf{c}$ or tail of $i$ - 11 KD \#2 tadpoles d, e, and sequences corresponding to il-11.L b, d or il-11.S c, e were PCR-amplified using P1U.L/P1D.L b, P1U.S/P1D.S c, P2U.L/P2D.L d, or P2U.S/P2D.S e primers. The PCR product of the expected length corresponding to deletion of the region between two gRNA target sites was sequenced. Blue characters: reference genomic sequences nearby gRNA target sites, red characters: mismatches from reference genomic sequences, bars: gaps, arrowheads: predicted cleavage sites. Numbers at the left corner indicates ratio of clones containing the sequence to total clones sequenced. $\mathbf{f}-\mathbf{i}$, Representative images of tails of control cas 9 mRNA-injected $\mathbf{f}$, il-11 KD \#1 $\mathbf{g}$, or il-11 KD \#2 $\mathbf{h}$ tadpoles at $7 \mathrm{dpa}$, and the tail regeneration length $\mathbf{i}$ are shown. Dark-field images $\mathbf{f}-\mathbf{h}$ are shown. Anterior is to the left, dorsal is up. White broken lines indicate amputated plane. Scale bars: $1 \mathrm{~mm}$. Regeneration length was normalised by snout to vent length (SVL). Box plots are inserted in the panels. Bars in the boxes represent median, upper and lower limits of the boxes represent the first and third quartiles, and whiskers represent maximum and minimum values. ${ }^{\star} P<0.05$, Dunnett's test 
(24, 72 and $120 \mathrm{hpa})$. il-11 expression was maintained for at least $120 \mathrm{hpa}$ (Fig. 1b), suggesting that $i l-11$ is also related to some late events after tail amputation.

Whole-mount in situ hybridisation (WISH; Fig. 1c-f, Supplementary Fig. 1) revealed no significant il-11 expression at
$0 \mathrm{hpa}$ at the amputated tail stumps, coinciding with the qRT-PCR results. Significant $i l-11$ expression was detected at the outer edge of the notochord and spinal cord near the amputation plane at 5 and $24 \mathrm{hpa}$, indicating that $i l-11$ expression was restricted to the amputation plane at the early phase of tail regeneration. At 72

a

\begin{tabular}{|ccccc|}
$\begin{array}{c}\text { Modified tet- } \\
\text { responsive element }\end{array}$ & $\begin{array}{c}\text { il-11-p2a peptide-acgfp } \\
\text { or acgfp }\end{array}$ & $\begin{array}{c}\text { Minimal } \\
\text { CMV } \\
\text { promoter }\end{array}$ & $\begin{array}{c}\text { Tet-on advanced } \\
\text { transactivator }\end{array}$ & \\
& & & & \#1U.LS \\
\hline
\end{tabular}

b

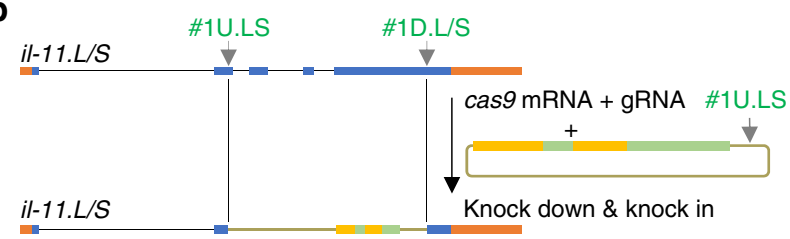

C

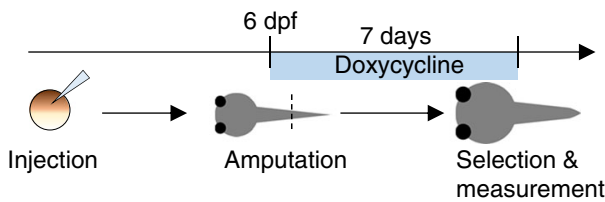

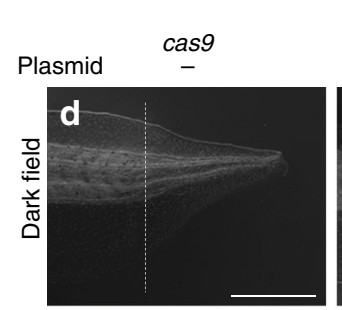
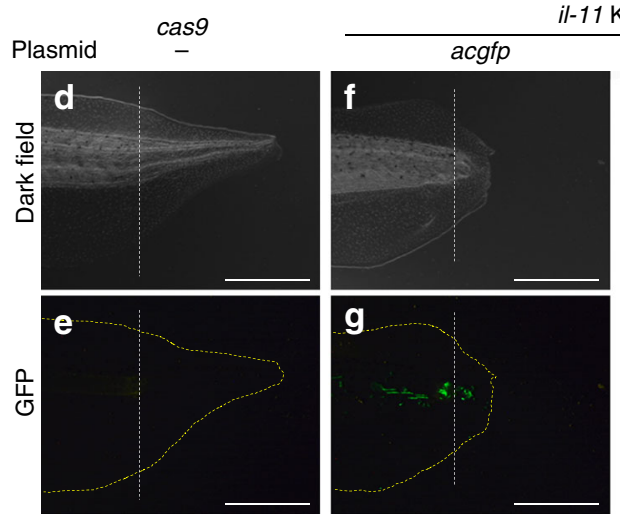

k

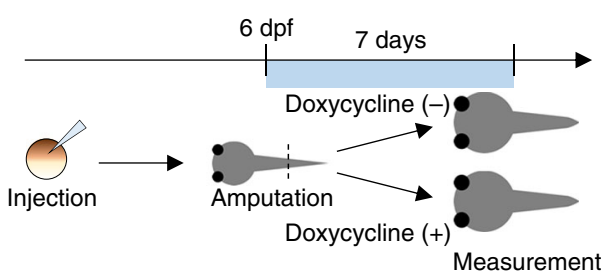

il-11 j
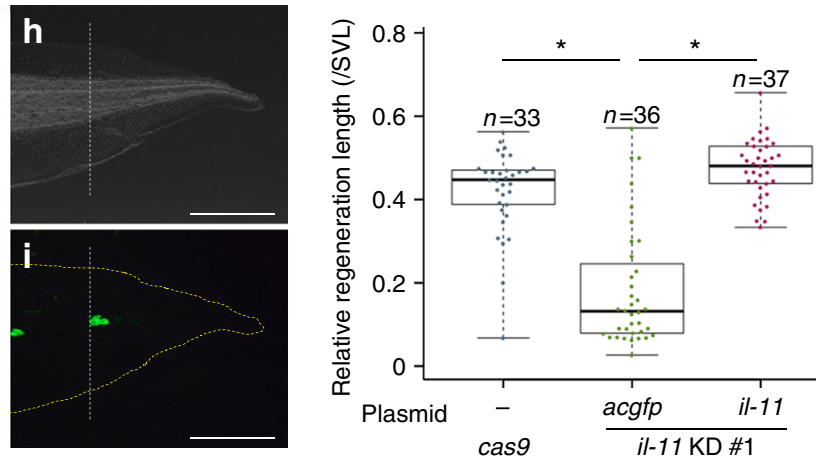
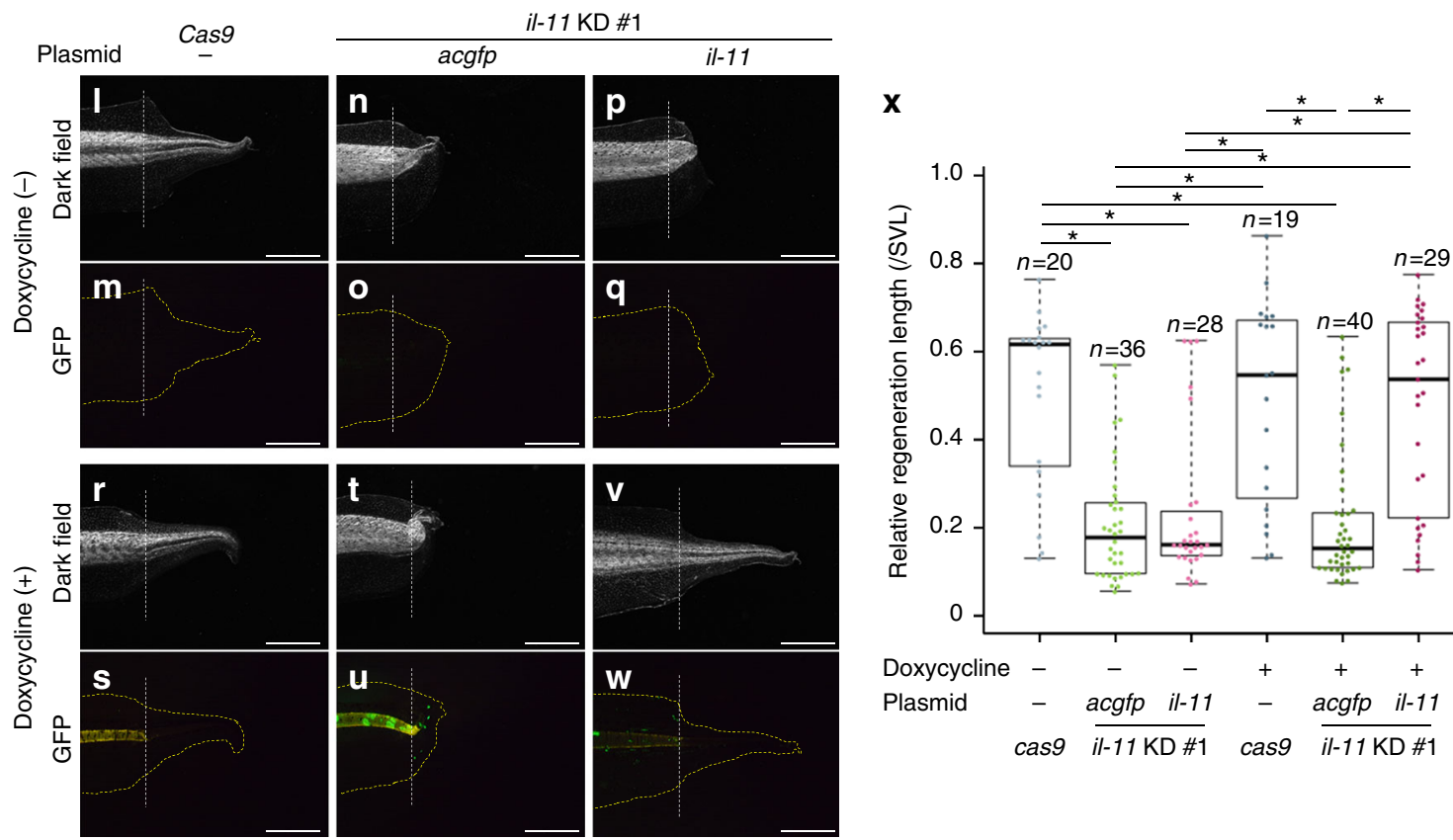
and $120 \mathrm{hpa}$, il-11 expression was detected at the tip of the regenerating tails, where the tail blastema cells are located (Fig. 1e, f). We previously observed that proliferating tail blastema cells begin to accumulate at the tip of the regenerating tails 3 days post amputation $(\mathrm{dpa})^{4}$. Induction of $i l-11$ expression at the amputated tail stumps in the early phases (5 and $24 \mathrm{hpa}$ ) and its suspension at the tip of regenerating tails in the later phases ( $>3 \mathrm{dpa}$ ) led us to hypothesise that $i l-11$ is involved in the induction and/or maintenance of tail blastema cells, which are undifferentiated progenitor cells of various tissues in the tail.

To investigate whether $i l-11$ is selectively expressed among IL-6 family members, we performed qRT-PCR for il- 6 and lif, which encode IL- 6 family members important for the differentiation or maintenance of stem/progenitor cells ${ }^{14-16}$. Expression of these two genes was modestly induced (at most, less than approximately threefold) after tail amputation compared to the expression of il-11 (Supplementary Fig. 2a-d), suggesting that $i l-11$ has a specific function in tail regeneration among IL-6 family members.

To investigate whether IL-11 signalling is actually activated, we visualised activation of Stat3, which is the major signalling molecule and transcription factor downstream of IL-11 10,11 , by immunohistochemistry using an antibody against phosphorylated Stat3. We found that phosphorylated Stat3 co-localised with the nuclei in notochord bud, spinal cord ampulla and epithelium in blastema of 72-hpa tadpoles (Supplementary Fig. 3). Although Stat 3 is activated by several upstream ligands, including IL- 6 and Lif, expression of these two ligands was not prominently elevated compared to that of $i l-11$ after tail amputation, as mentioned above, and thus it is plausible that the observed activation of Stat3 is mainly due to the expression of IL-11, and that IL-11 signalling is activated in various cell types in blastema.

To investigate whether IL-11-responsive cells are induced after tail amputation, we performed qRT-PCR for il11ra and il6st, which are genes for receptors of IL-11 ${ }^{6,7}$. Expression of these genes was modestly induced (at most, less than approximately threefold; Supplementary Fig. 2e-h) compared to the expression of $i l-11$, suggesting that the IL-11-responsive cells constitutively reside in intact tails and the number of the cells remains largely unaltered during the regeneration process.

il-11 is necessary for tail regeneration. We next examined whether $i l-11$ is necessary for tail regeneration by knocking-down il-11 using the CRISPR/Cas9 system. Because X. laevis has a long generation time of more than 1 year, we attempted to analyse the effect of $i l-11 \mathrm{KD}$ in $\mathrm{F}_{0}$ tadpoles. To efficiently knockdown $i l-11$ in $\mathrm{F}_{0}$ tadpoles, we designed guide RNAs (gRNAs) targeting two regions each in two $i l-11$ homoeologues of allotetraploid $X$. laevis, il-11.L and il-11.S (Fig. 2a), which is expected to cause a large deletion of the nucleotide sequences sandwiched by the two gRNA target sites, in addition to the frame shift that occurs at each of the two gRNA target sites of each gene. We co-injected these gRNAs (\#1U.LS, \#1D.L and \#1D.S) and cas 9 mRNA into fertilised eggs, and kept the hatched tadpoles till approximately stage $40-42$. We then amputated the tails of $\mathrm{F}_{0}$ tadpoles (il-11 KD \#1 tadpoles) at 6 days post fertilisation (dpf), and measured the regenerated tail length at $7 \mathrm{dpa}(13 \mathrm{dpf})$. To confirm that the observed phenotype was not due to an off-target effect, we also created another group of $\mathrm{KD}$ tadpoles (il-11 KD \#2 tadpoles) using another gRNA set targeting other sequences in il-11 (Fig. 2a), and compared the regenerated tail length of $i l-11 \mathrm{KD} \# 1$, \#2 and control cas 9 mRNA-injected tadpoles (cas 9 tadpoles). We detected a loss of genomic regions between the two gRNA target sites of il-11.L and il-11.S using PCR amplification of the interval regions (Fig. 2b-e), indicating that $i l-11$ genes could be edited by this approach. The ratio of normally developed il-11 KD tadpoles was not prominently lower than that of cas 9 tadpoles (Supplementary Table 1), suggesting that il-11 KD does not affect normal development under these conditions. The ratio of normally grown il-11 KD tadpoles after tail amputation was almost the same as that of cas9 tadpoles (Supplementary Table 1), suggesting that il-11 does not have a crucial role in wound healing or in immune responses after tail amputation. When we measured relative regenerated tail length normalised by snout to vent length (Supplementary Fig. $4 \mathrm{f}$ and $\mathrm{g}$ ), we found that the regenerated tail length was significantly shorter in both il-11 KD $\# 1$ and \#2 tadpoles compared to cas 9 tadpoles $(P<0.05$, Dunnett's test, Fig. 2f-i, Supplementary Fig. 4h), suggesting that il-11 is necessary for tail regeneration. Deletion of genomic regions between the two gRNA target sites of il-11.L and il-11.S was detected in the tail stump tissues of most of these tadpoles (Supplementary Fig. 4a-e), suggesting that the function of IL-11 was lost in at least some cells at the amputated tail stumps of most of these il-11 KD tadpoles.

To confirm that the shortened regeneration length was not due to a non-specific effect of chromosomal disruption, which might have been caused by genome editing, we performed a rescue experiment of the phenotype of $i l-11 \mathrm{KD}$ tadpoles. For this, an $i l-11$ rescue construct that expresses IL-11 with the expression marker AcGFP upon doxycycline treatment by the tet-on system (Fig. 3a), or a control construct that expresses AcGFP alone, was co-injected with cas 9 mRNA and gRNAs targeting il-11 into a one-cell stage embryo. In the $i l-11$ rescue and control acgfp-expressing constructs, the target sequence of gRNA \#1U. LS was inserted. Some constructs might be knocked into the il-11 gene locus by homology-independent double-strand break repair ${ }^{19}$ when the genomic $i l-11$ locus and the target sequence on the rescue construct were cleaved concurrently by the CRISPR/Cas9 system (Fig. 3b). In addition to the free constructs remaining in the cells of the tadpole tail at the time of tail amputation, the knocked-in constructs would facilitate the expression of il-11 by stably inserting into the genome. The injected tadpoles were kept till approximately stage $40-41$, then their tails were amputated at $6 \mathrm{dpf}$ following doxycycline

Fig. 3 Rescue experiment of the phenotype of il-11 KD tadpoles. a Schematic drawing of a rescue construct. Yellow boxes represent cis-regulatory elements, and green boxes represent coding sequences. gRNA \#1U.LS target site was inserted in the construct. In the control construct, acgfp was inserted instead of il-11-p2a peptide-acgfp. b Schematic drawing of knock-in and knockdown. Bars: introns, orange boxes: untranslated regions, blue boxes: coding sequences, dark grey arrows: gRNA target sequences. c Schematic drawing of a rescue experiment. $\mathbf{d}$-j Representative images of tails of control cas 9 mRNA-injected $\mathbf{d}$, e, il11 knockeddown acgfp-expressing $\mathbf{f}$, $\mathbf{g}$, or il-11 knockeddown il-11-expressing $\mathbf{h}$, $\mathbf{i}$ tadpoles, and their regeneration length $\mathbf{j}$ are shown. $\mathbf{k}$, Schematic drawing of a rescue experiment using tadpoles treated with or without doxycycline. I-x Representative images of tails of untreated cas 9 mRNA-injected $\mathbf{I}$, $\mathbf{m}$, il-11 knockeddown acgfp-expressing $\mathbf{n}$, o, or il-11 knockeddown il-11-expressing $\mathbf{p}$, q tadpoles, or doxycycline-treated cas 9 mRNA-injected $\mathbf{r}$, s, il-11 knockeddown acgfp-expressing $\mathbf{t}$, $\mathbf{u}$, or il-11 knockeddown il-11-expressing $\mathbf{v}, \mathbf{w}$ tadpoles and their regeneration length $\mathbf{x}$ are shown. Dark-field images $\mathbf{d}, \mathbf{f}, \mathbf{h}, \mathbf{I}, \mathbf{n}, \mathbf{p}, \mathbf{r}, \mathbf{t}, \mathbf{v}$ and GFP2-filtered images $\mathbf{e}, \mathbf{g}, \mathbf{i}, \mathbf{m}, \mathbf{0}, \mathbf{q}, \mathbf{s}, \mathbf{u}, \mathbf{w}$ are shown. Regeneration length was normalised by snout to vent length (SVL). Box plots are inserted in the panels. Bars in the boxes represent median, upper and lower limits of the boxes represent the first and third quartiles, and whiskers represent maximum and minimum values. Scale bars: $1 \mathrm{~mm}$. Anterior is to the left, dorsal is up. Yellow broken lines indicate outline of the tails. White broken lines indicate amputated plane. ${ }^{\star} P<0.05$, Tukey-Kramer's test 
treatment, and their regenerated tail length was measured at 7 dpa (13 dpf; Fig. 3c, Supplementary Fig. 5a, b). To select tadpoles in which the constructs were successfully introduced, normally grown tadpoles with GFP signal at the amputation plane were selected under a fluorescence stereomicroscope for measurement of the regeneration length of tadpoles with forced acgfp or il-11 expression (Supplementary Table 2). In the cas 9 group, all normally grown tadpoles were selected, because cas 9 tadpoles do not show GFP signals (Supplementary Table 2). Although the relative regenerated tail length of il-11 KD acgfp-expressing
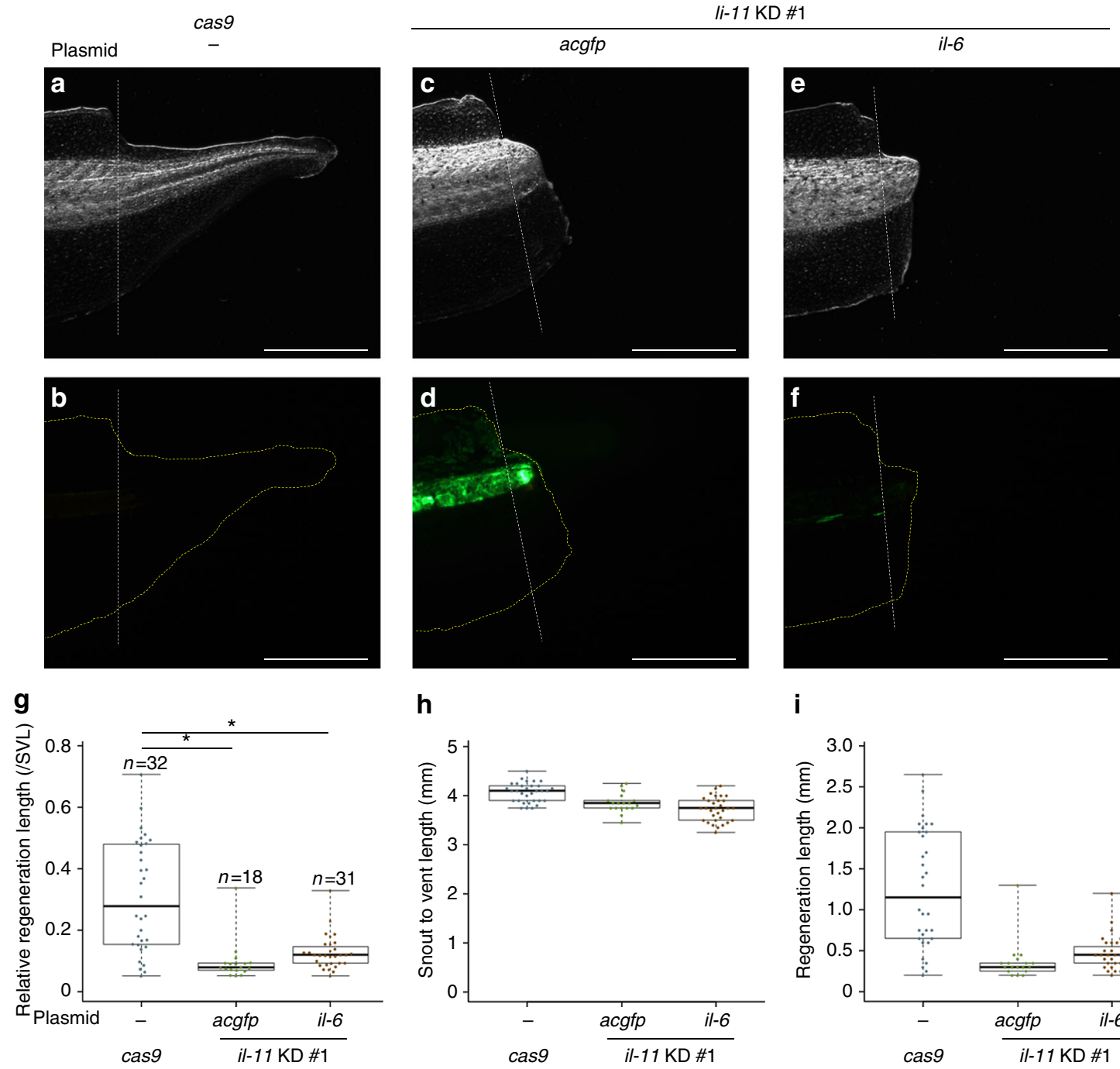

h

\section{i}
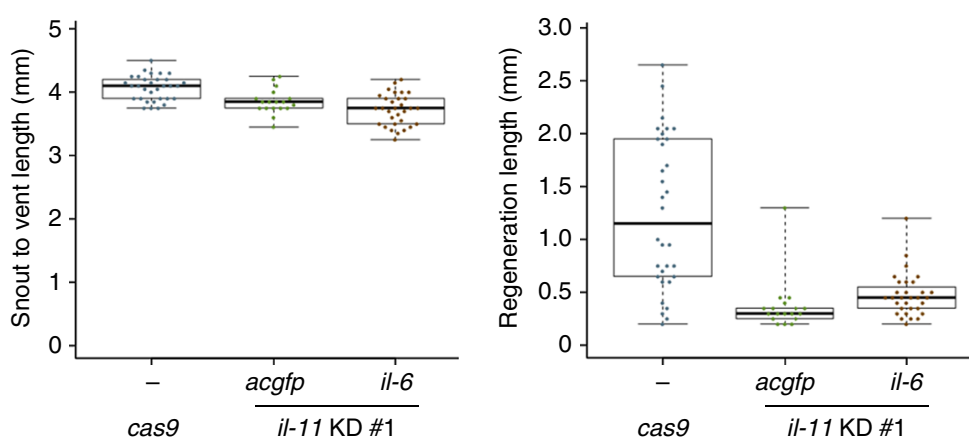

j

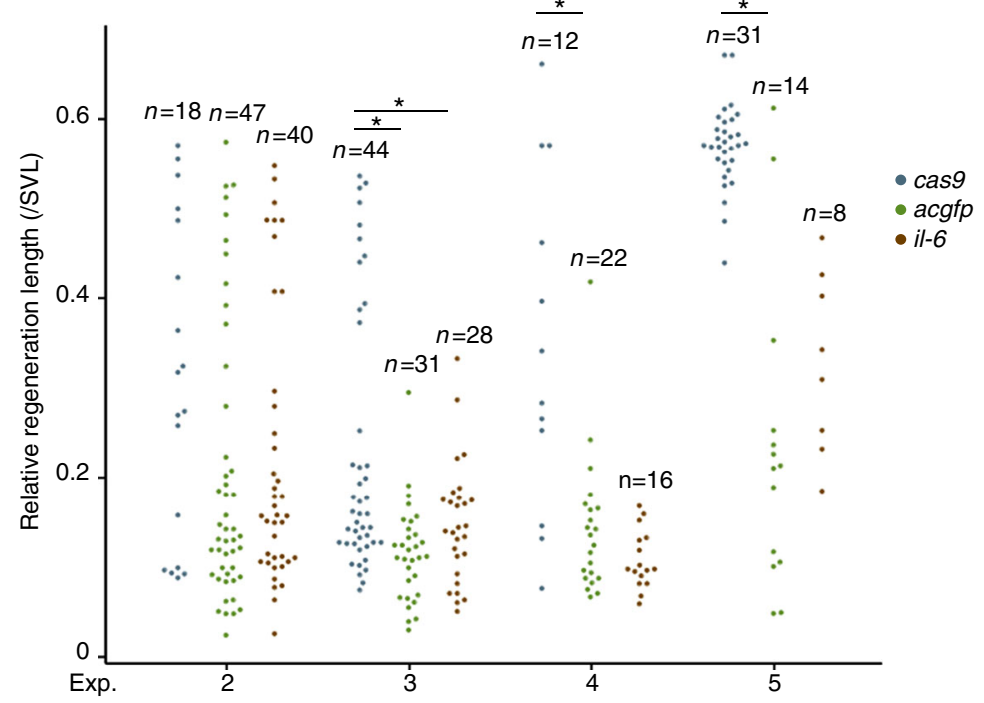


construct-introduced tadpoles was significantly shorter compared to cas 9 mRNA-injected tadpoles, the tail shortening was almost completely rescued in il-11-rescued tadpoles $(P<0.05$, Tukey - Kramer's test, Fig. $3 \mathrm{~d}-\mathrm{j}$, Supplementary Fig. $5 \mathrm{c}$ ), suggesting that $i l-11$ is necessary for tail regeneration.

We also investigated whether the rescue effect is specific for il-11 among the IL-6 family members by forcing the expression of il-6 in il-11 KD tadpoles. Normally grown tadpoles with GFP signal at the amputation plane were selected for measurement of the regeneration length (Supplementary Table 3). Regeneration length was almost the same in il-11 KD tadpoles with forced expression of $i l-6$ compared to $i l-11 \mathrm{KD}$ tadpoles expressing acg $f p$ (Fig. 4), suggesting that signalling pathways specific to il-11 among IL-6 family members are necessary for tail regeneration.

To further confirm that the rescue effect of $i l-11$ is not due to the introduced construct itself, but to the expression of $i l-11$, we compared the regeneration length of doxycycline-treated and untreated tadpoles. Injected tadpoles were divided into two groups after tail amputation at $6 \mathrm{dpf}$ : the first group was maintained in $0.1 \times$ Steinberg's solution and the second group was maintained in $0.1 \times$ Steinberg's solution with $50 \mu \mathrm{g} / \mathrm{ml}$ of doxycycline, and regeneration length was measured at $7 \mathrm{dpa}$ (Fig. 3k). The ratio of normally grown tadpoles after tail amputation was not prominently different between the doxycycline-treated and untreated groups (Supplementary Table 4), suggesting that doxycycline treatment did not affect tadpole viability under these conditions. The regeneration length of all normally developed tadpoles was measured and normalised by the snout to vent length (Supplementary Fig. 5d, e). GFP signals were very weak in untreated acgfp- and il-11-expressing construct-introduced tadpoles compared to their doxycyclinetreated counterparts (Fig. 3o, q, u, w), suggesting that the expression of AcGFP and IL-11 is doxycycline-dependent. In the untreated group, regeneration length was significantly shorter both in acgfp-and il-11-expressing construct-introduced tadpoles compared to cas 9 tadpoles (Fig. 31-q, x, Supplementary Fig. 5f). In the doxycycline-treated group, however, regeneration length was significantly shorter in acgfp-expressing construct-introduced tadpoles compared to il-11-expressing construct-introduced and cas 9 tadpoles, and the regeneration length of il-11-expressing construct-introduced tadpoles was almost the same as that of cas 9 tadpoles $(P<0.05$, Tukey-Kramer's test, Fig. 3r-w, $\mathrm{x}$, Supplementary Fig. 5f), clearly indicating that $i l-11$ is necessary for tail regeneration.

il-11 induces and maintains progenitors of several tissues. Next, to analyse the role of $i l-11$ in tail regeneration, we compared gene expression profiles between the amputated tail stumps of il-11 KD and cas 9 tadpoles. When we compared the morphology of regenerating tails of $i l-11 \mathrm{KD}$ and cas 9 tadpoles every day after tail amputation, they were almost the same until $2 \mathrm{dpa}$. At $3 \mathrm{dpa}$, however, cas 9 tadpoles showed prominent growth of the regenerated tail, whereas il-11 KD tadpoles showed only moderate growth (Supplementary Fig. 6), suggesting that some regenerative processes present between 2 and 3 dpa were disrupted in il-11 KD tadpoles. Therefore, we compared gene expression profiles in tail stump tissues of $i l-11 \mathrm{KD} \# 1$ and cas 9 tadpoles at 2 dpa by RNA-sequencing.

We found that among top 10 genes which showed highest upregulation in il-11 KD \#1 tadpoles, five genes are reported to be selectively expressed in skeletal muscle in mouse (Fig. 5a and Supplementary Fig. 7), suggesting that ratio of mature tissues were relatively high in tail stump tissues of il-11 KD tadpoles compared to cas 9 tadpoles. This prompted us to hypothesise that ratio of undifferentiated cells were relatively high in tail stump tissues of cas 9 tadpoles compared to $i l-11 \mathrm{KD}$ tadpoles. Then we found that the expression levels of several undifferentiated cell markers were affected by $i l-11$ knockdown (Fig. 5a, b). In il-11 KD \#1 tadpoles compared to cas 9 tadpoles, we observed a tendency toward or significant (adjusted $P<0.05$, Wald test) downregulation of notochord homeobox (not), an early notochord marker in Xenopus ${ }^{20,21}$; doublecortin $(d c x)$, a marker for a subpopulation of muscle progenitor cells in mouse ${ }^{22,23}$; and runt related transcription factor 1 (runx1), a marker of progenitor cells of Rohon-Beard sensory neurons ${ }^{24}$, which are a subpopulation of primary sensory neurons located in the dorsal spinal cord that mediate the response to touch in larval stages of lower vertebrates.

When we performed WISH (Fig. 5c-h, Supplementary Fig. 8), not was expressed at the tip of the notochord bud, $d c x$ was expressed in the muscle near the amputation plane, and runx1 was expressed in the spinal cord ampulla and ventral side of the blastema. The ratio of tadpoles with strong expression of the genes tended to be higher in the cas 9 group (not: 10/10, $d c x: 10 / 10$, and runx1: 9/10) compared to the il-11 KD \#1 and \#2 groups (not: $4 / 10$ and 1/10; $d c x: 3 / 10$ and 3/10; runx1: $6 / 10$ and 7/10). These results suggest that induction and/or maintenance of the progenitor cells of the notochord, muscle, or Rohon-Beard sensory neurons are impaired in il-11 KD tadpoles.

We also performed section ISH for the undifferentiated marker genes using doxycycline-treated cas 9 mRNA-injected, acgfp- or il-11-expressing il-11 KD tadpoles fixed at $2 \mathrm{dpa}$. We detected signals for not and $d c x$. Expression of not was detected in the notochord bud, and expression of $d c x$ was detected in the muscle and spinal cord (Supplementary Fig. 9). The ratio of tadpoles with strong expression in the notochord bud (not) or muscle $(d c x)$ tended to be higher in $\operatorname{cas} 9$ (not: 6/6, $d c x: 11 / 11)$ and il-11-rescued (not: 6/6. dcx: 11/11) tadpoles compared to control acgfp-expressing il-11 KD tadpoles (not: $2 / 6, d c x: 3 / 11$ ), further suggesting that induction and maintenance of undifferentiated cells are il-11-dependent. The expression of $d c x$ in the spinal cord seems to be il-11 independent, because signals for $d c x$ in the spinal cord were observed in tadpoles in all groups.

il-11 can induce progenitor cells in intact tail. Finally we examined whether expression of $i l-11$ is sufficient for the induction and/or maintenance of the progenitor cells even in intact tails. For this, we co-injected acgfp- or il-11-expressing vector with cas 9 mRNA and gRNA targeting the tyrosinase locus, which is the gene responsible for albinism and unnecessary for tadpole survival, into the one-cell stage embryo to facilitate the

\footnotetext{
Fig. 4 il- 6 does not rescue the shortened regeneration length of il-11 KD tadpoles. a-f Representative images of tails of control cas 9 mRNA-injected $\mathbf{a}$, $\mathbf{b}$, il- 11 knockeddown acgfp-expressing $\mathbf{c}$, $\mathbf{d}$, or il-11 knockeddown il-6-expressing $\mathbf{e}, \mathbf{f}$ tadpoles, and their regeneration length $\mathbf{g}$ are shown. Dark-field images $\mathbf{a}$, $\mathbf{c}$, $\mathbf{e}$ and GFP2-filtered images $\mathbf{b}, \mathbf{d}, \mathbf{f}$ are shown. Regeneration length was normalised by snout to vent length (SVL). $\mathbf{h}, \mathbf{i}, \mathrm{SVL} \mathbf{h}$ and regeneration length $\mathbf{i}$ used for calculation in $\mathbf{g}$ are shown. $\mathbf{j}$ Biological replicates using different batches of tadpoles are shown. Box plots are inserted in the panels. Bars in the boxes represent median, upper and lower limits of the boxes represent the first and third quartiles, and whiskers represent maximum and minimum values. Scale bars: $1 \mathrm{~mm}$. Anterior is to the left, dorsal is up. Yellow broken lines indicate outline of the tails. White broken lines indicate amputated plane. ${ }^{\star} P<0.05$, Tukey-Kramer's test
} 
a

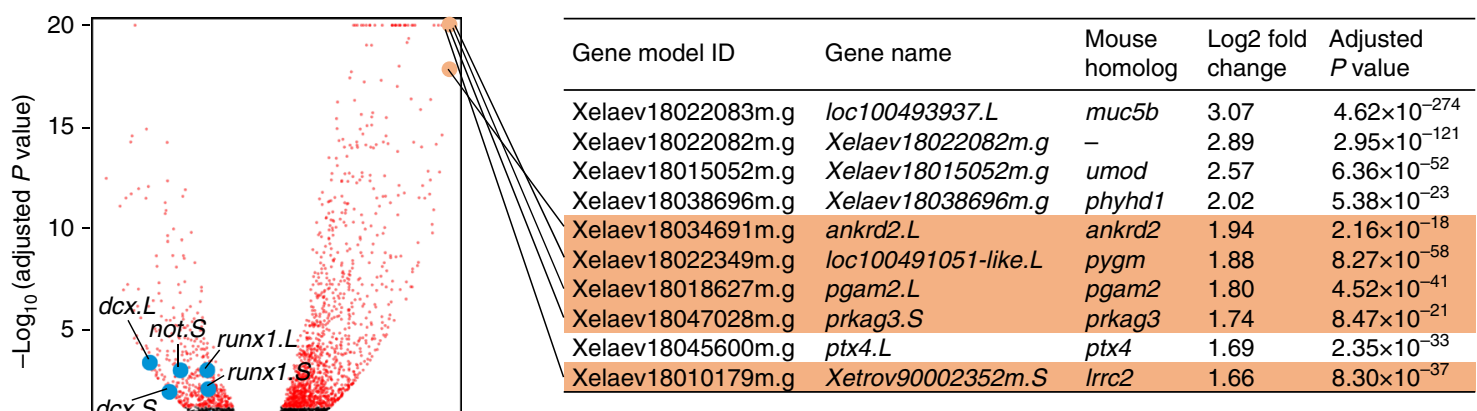

$\begin{array}{lll}1 & 1 & 0\end{array}$

$\log _{2}$ fold change (il-11 KD \#1/cas9)

b

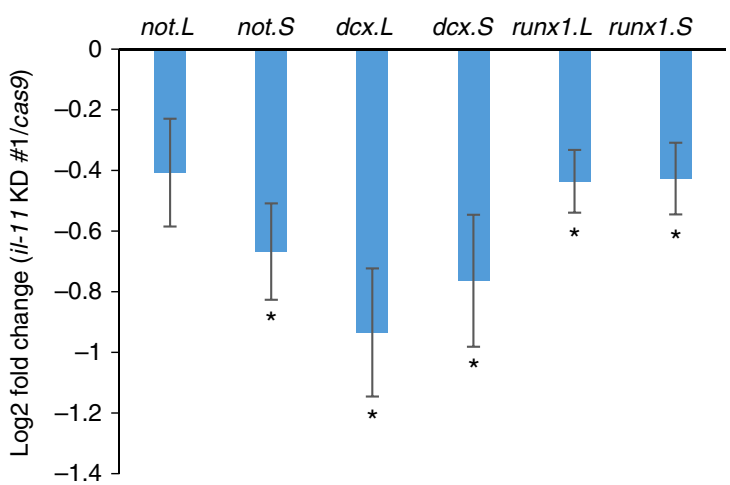

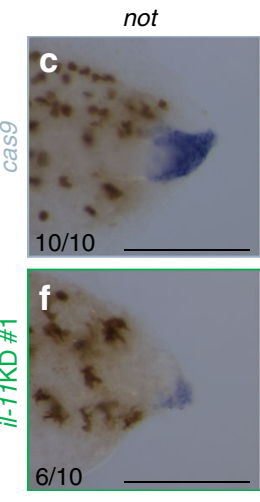

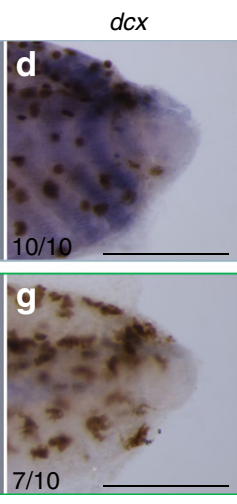

runx1

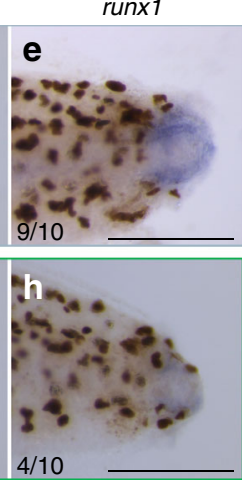

Fig. 5 il-11 induces or maintains tissue progenitor cells across cell lineages during tail regeneration. a Gene expression profile of $i$ - 11 KD \#1 and cas9 tadpoles. Left panel: volcano plot. Fold-change of expression level of il-11 KD \#1 tadpoles over control tadpoles measured by RNA-sequencing are plotted on horizontal axis in $\log _{2}$ scale, and adjusted $P$-values are plotted on vertical axis in - $\log _{10}$ scale. Genes with significant fold-changes (adjusted $P<0.05$ ) are plotted in red. Points beyond the range are plotted on the edge of the graph. Representative undifferentiated markers are plotted in blue. Right panel: top 10 genes which showed highest upregulation in il-11 KD \#1 are listed. Mouse homologues of genes indicated in orange are reported to be selectively expressed in skeletal muscle in mice (Supplementary Fig. 7). These genes correspond to orange plots in the left panel. b Fold-change of expression levels of representative undifferentiated markers measured by RNA-sequencing. Mean \pm s.e.m. $n=3$. *adjusted $P<0.05$, Wald test. c-h, Localisation of undifferentiated markers. Representative WISH images for not $\mathbf{c}, \mathbf{f}, d c x \mathbf{d}, \mathbf{g}$, or runx $\mathbf{e}$, $\mathbf{h}$ in control $\mathbf{c}-\mathbf{e}$ or il-11 KD \#1 tadpoles $\mathbf{f}-\mathbf{h}$ are shown. Anterior is to the left, dorsal is up. Blue/purple colour represents signals for the genes. Brown pigments are melanophores of the tadpoles. Scale bars: $500 \mu \mathrm{m}$. Numbers at the bottom corner indicate the total ratio of tadpoles showing the corresponding expression pattern from two batches

expression of $i l-11$ by construct knocked into the tyr locus, as well as free plasmid retained in the cells of the tadpole tails. Then, the injected tadpoles at $5 \mathrm{dpf}$ (approximately stage 40-42) were treated with doxycycline for 2 days, and the expression levels of the undifferentiated cell marker genes in their tails were compared (Fig. 6a-g). Although not expression was scarcely detected, or detected at very low levels in acgfp-expressing tails, it was detected in il-11-expressing tails (Fig. 6h, Supplementary Fig. 10a). $d c x$ was significantly upregulated in il-11-expressing tails compared to acgfp-expressing tails $(P<0.05$, Student's $t$-test, Fig. 6i, Supplementary Fig. 10b). The expression level of runx1 tended to be higher in il-11-expressing tails than in acgfp-expressing tails (Fig. 6j, Supplementary Fig. 10c).

To investigate whether the number of cells expressing undifferentiated marker genes is increased by forced expression of $i l-11$, we performed section ISH. We detected signals for not and $d c x$. not signals were detected in cells in the notochord sheath of il-11-expressing tadpoles, whereas few signals were detected in acgfp-expressing cells (Fig. 6k-m, Supplementary Fig. 11a). $d c x$ signals were detected in the spinal cord and muscle (Fig. 6n, o, Supplementary Fig. 11b). The ratio of muscle expressing $d c x$ was higher in il-11-expressing tadpoles compared to acgfp-expressing tadpoles (Fig. 6p), suggesting that IL-11 is sufficient to induce and maintain progenitor cells even in intact tails. Notably, the fact that the genes upregulated in il-11-expressing tails were undifferentiated cell markers of different tissues suggests that induction of IL-11 alone is sufficient to induce progenitor cells derived from multiple tissues in intact tail, without additional factors that are induced by tail amputation.

\section{Discussion}

In the present study, we first analysed the expression of il-11. il-11 was expressed in the spinal cord and notochord at the amputation plane early after tail amputation, and continued to be expressed in the tip of the spinal cord ampulla and notochord bud in later phases of regeneration (Supplementary Fig. 1g, h). These regions correspond to the area where runx1- or not-expressing cells reside (Supplementary Figs. 8k, m, o, q, 9a-c). Therefore, il-11 might induce and maintain these progenitor cells in an autocrine/paracrine manner.

Next, we investigated whether $i l-11$ is necessary for tail regeneration by creating $i l-11 \mathrm{KD} /$ rescue tadpoles. In il-11-rescued tadpoles, GFP signals were sparse (Fig. $3 \mathrm{i}, \mathrm{w}$ ). It is important to note that IL-11 is cleaved from AcGFP at the P2A 
a

\begin{tabular}{cccc|}
$\begin{array}{c}\text { Modified tet- } \\
\text { responsive element }\end{array}$ & $\begin{array}{c}\text { il-11-p2a peptide -acgfp } \\
\text { or acgfp }\end{array}$ & $\begin{array}{c}\text { Minimal } \\
\text { CMV } \\
\text { promoter }\end{array}$ & $\begin{array}{c}\text { Tet-on advanced } \\
\text { transactivator }\end{array}$ \\
\hline
\end{tabular}

b

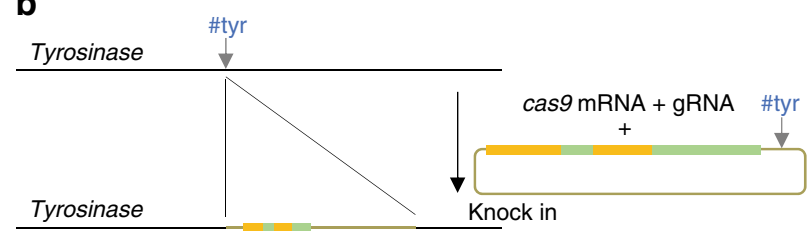

C

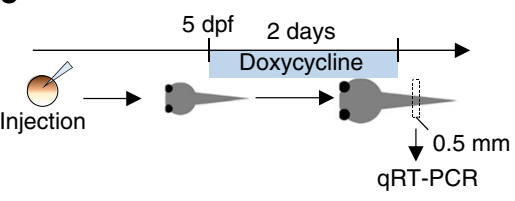

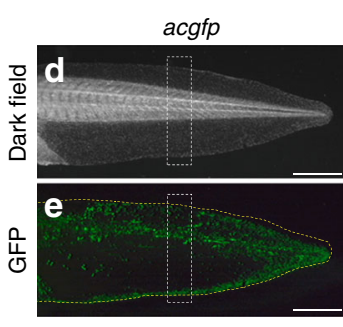
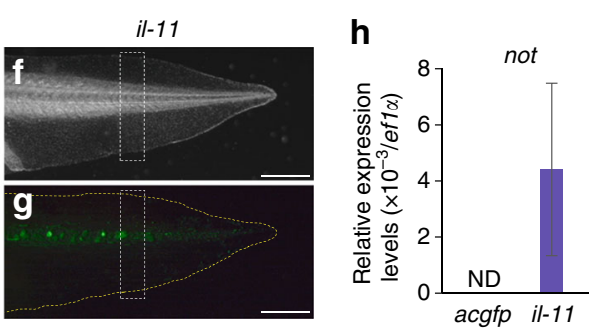
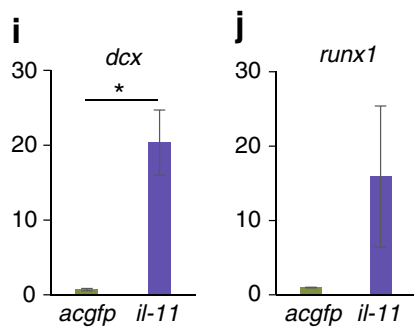

$d c x$

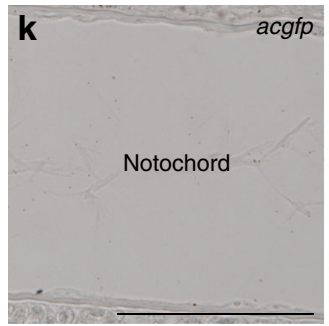

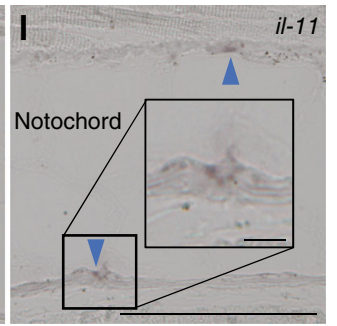
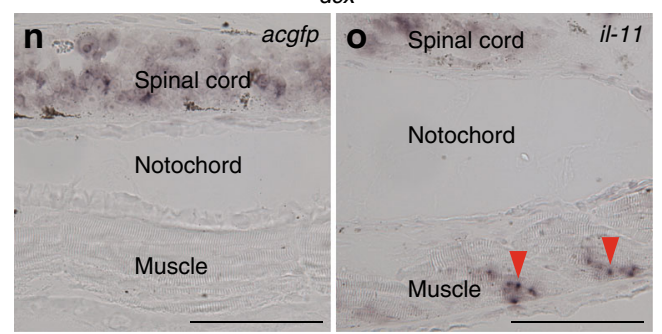

m

*

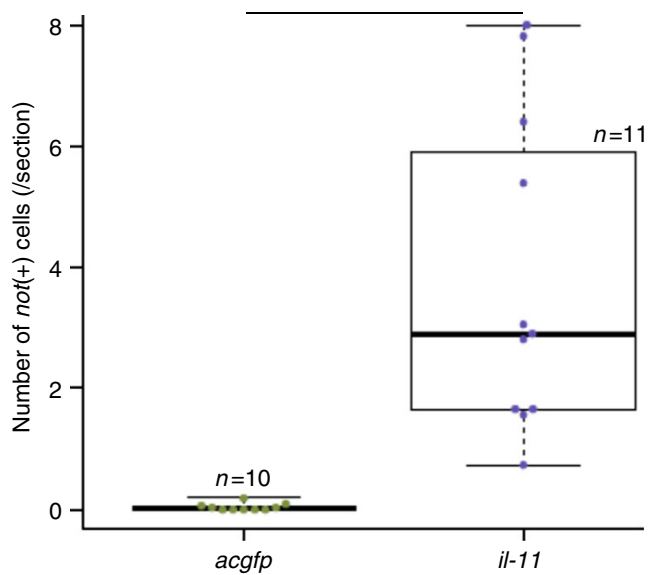

p

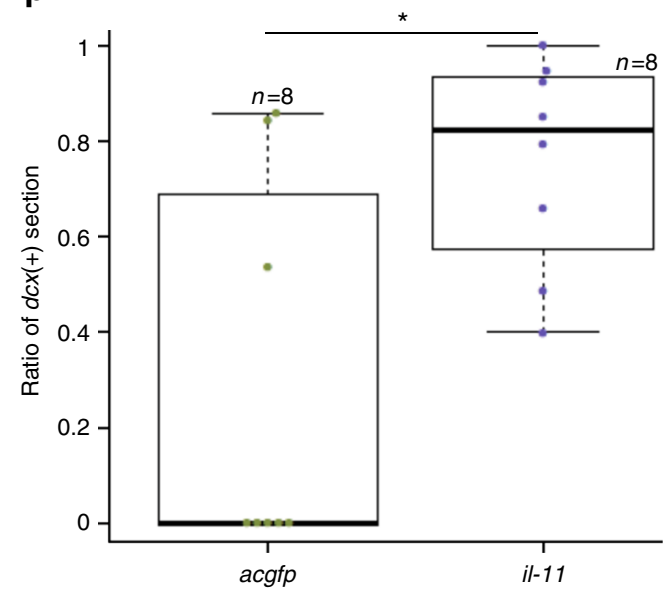

Fig. 6 il-11 is sufficient for induction of progenitor cells across cell lineages in intact tadpole tail. a Schematic drawing of a construct for forced expression of il-11. Yellow boxes represent cis-regulatory elements, and green boxes represent coding sequences. gRNA \#tyr target site was inserted in the construct. In the control construct, acgfp was inserted instead of il-11-p2a peptide-acgfp. b Schematic drawing of knock-in. Dark grey arrows: gRNA \#tyr target sequences. c Schematic drawing of a gain of function experiment. $\mathbf{d}-\mathbf{g}$, Representative images of tadpoles used in this experiment. acgfp-d, e or il-11-f, $\mathbf{g}$ expressing tails 2 days after doxycycline treatment are shown. Tail tissues indicated in white broken lines from approximately 10 tadpoles were used for qRT-PCR. Dark-field images $\mathbf{d}, \mathbf{f}$ and GFP2-filtered images $\mathbf{e}, \mathbf{g}$ are shown. Scale bars: $1 \mathrm{~mm}$. Anterior is to the left, dorsal is up. Yellow broken lines indicate outline of the tails. $\mathbf{h}-\mathbf{j}$, Expression levels of not $\mathbf{h}, d c x \mathbf{i}$, or runx $\mathbf{j}$ in tails expressing acgfp or il-11 were measured by qRT-PCR. Vertical axes represent relative expression levels normalised by those of ef1 $\alpha$. Mean \pm s.e.m. $n=3$. $\mathbf{k}, \mathbf{I}, \mathbf{n}, \mathbf{0}$, Representative ISH images for not $\mathbf{k}$, I or $d c x \mathbf{n}, \mathbf{o}$ in acgfp $\mathbf{k}, \mathbf{n}$ or il-11 I, o-expressing intact tadpole tails 2 days after doxycycline treatment are shown. Several not-expressing cells in the notochord sheath (blue arrowheads) and dcx-expressing muscle (red arrowheads) were detected in il-11-expressing tails. $\mathbf{m}$ The number of not-expressing cells in the notochord sheath in all sections containing notochord was counted. $\mathbf{p}$ The ratio of sections containing $d c x$-expressing muscle in all sections containing muscle was calculated. Box plots are inserted in the panels. Bars in the boxes represent median, upper and lower limits of the boxes represent the first and third quartiles, and whiskers represent maximum and minimum values. Scale bars: $100 \mu \mathrm{m}$ except inset in (I) $(10 \mu \mathrm{m})$. Anterior is to the left, dorsal is up. Magenta/purple colour represents signals for the genes. Inset: magnified view of boxed area. ${ }^{\star} P<0.05$, Student's $t$-test. ND: not detected 
peptide, and therefore GFP signals reflect the localisation of not IL-11 itself, but of il-11-expressing cells. One possible explanation for how the sparse expression of $i l-11$ could rescue the shortened regeneration length is that IL-11 was very strongly expressed in the source cells and secreted everywhere in the tail at a high concentration, and IL-11-responsive cells that express IL-11 receptors at the tip of the blastema received the IL-11 signal. The result that the forced expression of $i l-11$ in the intact tail prominently upregulated expression of undifferentiated marker genes (Fig. 6) supports the idea that the concentration of IL-11 is sufficient for undifferentiated cells to respond even though the GFP signal was sparse. Because we did not observe additional tails or mass in the tails with forced $i l-11$ expression, it is possible that additional signals at the tip of the blastema are needed for prolonged survival or continued proliferation of the progenitor cells.

We then analysed the role of $i l-11$ in tail regeneration. We found that marker genes of progenitor cells in different cell lineages were downregulated in il-11 KD tadpoles (Fig. 5), and upregulated in tadpoles with forced il-11 expression (Fig. 6). Notably, these marker genes were not exclusively expressed in a single cell population. $d c x$ was expressed in the spinal cord (Fig. 6n, o, Supplementary Figs. 8l, n, p, 9e-h, 11b) as well as in muscle. $d c x$ is expressed in neuronal precursor cells in the developing mouse brain ${ }^{25}$. Therefore, it is possible that these cells are neural progenitors, although they might be il-11 independent, because the signal for $d c x$ in the spinal cord was not prominently downregulated in il-11 KD tadpoles (Supplementary Figs. 8n, p, $9 \mathrm{~h}$ ), or upregulated in tadpoles with forced expression of il-11 (Fig. 6n, o, Supplementary Fig. 11b). runx1 was expressed in the ventral region of the amputated tail stumps in addition to the spinal cord (Supplementary Fig. 8m, o, q). runx 1 is expressed in hemogenic endothelium in mice ${ }^{26}$. The dorsal aorta is located at the ventral side of the Xenopus tadpole tail. Therefore, runx1 might also be expressed in the postulated hemogenic endothelium at the amputated plane in Xenopus. Further studies, such as lineage tracing of cells expressing the markers or transplantation analysis of the progenitors will more strongly support the idea that the cells induced and maintained by $i l-11$ are actually progenitor cells of various cell lineages.

Although previous studies reported that $i l-11$ is expressed in regenerating heart in zebrafish ${ }^{18}$, and that IL-11 maintains expression of undifferentiated markers in human embryonic stem cells $^{17}$, our findings are the first to indicate that IL-11 plays a key role in the induction and maintenance of the progenitor cells of various tail tissues. Downstream of IL-11, it is probable that some intracellular signals, which have been investigated in mammals ${ }^{5}$, function to maintain a broad range of stem/progenitor cells in an undifferentiated state. Analysing the molecular mechanisms that link tail amputation to $i l-11$ induction, such as the identification of a regeneration-specific enhancer element, which is suggested in zebrafish ${ }^{27}$, will unveil regeneration-specific molecular and cellular processes that start after amputation in organ regeneration.

\section{Methods}

Statistical tests were not used to pre-determine sample size. The experiments were not randomised or blinded. Normally developed tadpoles were used in the experiments.

Animals and tail amputation. Animals were obtained and treated as described previously ${ }^{4}$. In brief, tadpoles were obtained by mating wild-type Xenopus laevis, and maintained in $0.2 \%$ salt water at $20^{\circ} \mathrm{C}$ until they were used. Tadpole tails were amputated at 5 days post fertilisation (dpf) (Nieuwkoop and Faber stage ${ }^{28}$ 39-42) after anaesthetisation with $0.02 \%$ MS-222 (Sigma-Aldrich, St. Louis, MO). We followed the Guidelines for Proper Conduct of Animal Experiments of Science Council of Japan. The protocol in this study was approved by the Committee on the Ethics of Animal Experiments of the Graduate School of Science at the University of Tokyo.

qRT-PCR. qRT-PCR was performed essentially as described previously ${ }^{4}$ with mino modifications. In brief, total RNA was extracted using RNeasy mini kit (Qiagen, Germany), and reverse transcribed using PrimeScript RT reagent Kit with gDNA Eraser (Perfect Real Time; TaKaRa, Japan). Targets were amplified using SYBR premix ExTaq II (Tli RNaseH plus; TaKaRa) and a LightCycler 480 Real-time PCR System (Roche Diagnostics, Switzerland). The amounts of the transcripts were normalised with those of elongation factor-1 alpha (efla). The forward and reverse primers used for amplification of each gene were as follows: ef $1 \alpha$; 5'-GGAACGGTGACAACATGC-3' and 5'-AGGCAGACGGAGAGGCTTA-3', $i l-11 ; 5^{\prime}$-TCCTGAAGCTAAGCACTGACCT-3' and $5^{\prime}$-TGAATTCCGTTAAATT CGTGGTCCA-3', il-6; 5'-TTGTGGCCACTCACCTTGGT-3' ${ }^{\prime}$ and $5^{\prime}$-GATGAGC TTCGCTAGGGCCA-3', lif; 5'-AACAGCGATGCCATTGGACA-3' and 5'-TGCT CCAGATGCCAGCTCTT-3', ill 1ra; 5'-AATCACATCGCTGGCGTGTG-3' and $5^{\prime}$-CATCCAGCAGCCGGAAACTG-3', il $6 s t$; 5' -CCAGAAGCTCCACCTTC CAG- $3^{\prime}$ and $5^{\prime}$-CCATTGGCTACGGAGTCACTT- $3^{\prime}$, not $; 5^{\prime}$-GAACAGACAGAC CTGCCTCC- $3^{\prime}$ and $5^{\prime}$-AGCGGTAGGGCATAGATGGG-3' ${ }^{\prime} d c x ; 5^{\prime}$-TGCCGATT CAGCCAATGGAA- ${ }^{\prime}$ ' and $5^{\prime}$-TGCTTACGAAGGCTTCCTGG-3', runx 1 ; 5' ${ }^{\prime}$-CAG CCCGCATCACCCAAATC-3' and 5' ${ }^{\prime}$-TCTGACCCTGAGGCTGAGGA-3'.

WISH. WISH was performed as described previously ${ }^{4}$. In brief, whole body of the tadpoles were fixed with MEMFA (50 mM 3-(N-morpholino) propanesulfonic acid, $\mathrm{pH} 7.4$, including $2 \mathrm{mM}$ ethylene glycol-bis(2-aminoethylether)- $\mathrm{N}, \mathrm{N}, \mathrm{N}^{\prime}, \mathrm{N}^{\prime}$-tetraacetic acid, $1 \mathrm{mM} \mathrm{MgSO}_{4}, 3.7 \%$ formaldehyde) fixative, and kept in methanol at $-20{ }^{\circ} \mathrm{C}$ until they were stained. Then they were rehydrated, treated with $50 \mu \mathrm{g} / \mathrm{ml}$ of proteinase $\mathrm{K}$ for $10 \mathrm{~min}$, treated with $0.5 \%$ of acetate anhydride in $0.1 \mathrm{M}$ of triethanolamine, and fixed with $3.7 \%$ formaldehyde. The specimens were

pre-hybridised in the hybridisation buffer (Denhardt's solution (Wako, Japan) including $50 \%$ formamide, $0.75 \mathrm{M} \mathrm{NaCl}, 0.075 \mathrm{M}$ trisodium citrate, $100 \mu \mathrm{g} / \mathrm{ml}$ heparin, $0.1 \%$ Tween $20,0.1 \%$ 3-((3-Cholamidopropyl) dimethylammonio) propanesulfonate, and $1 \mathrm{mg} / \mathrm{ml}$ Ribonucleic acid from torula yeast Type VI (Sigma-Aldrich)) for $4-6 \mathrm{~h}$ at $60^{\circ} \mathrm{C}$, and hybridised with the probe solution $(1 \mu \mathrm{g} / \mathrm{ml}$ of digoxigenin labelled RNA probe in hybridisation buffer) for 4 nights at $60^{\circ} \mathrm{C}$. They were treated with RNase solution (1\% RNase Cocktail (Invitrogen, Waltham, MA) in $0.3 \mathrm{M} \mathrm{NaCl}$ and $0.03 \mathrm{M}$ trisodium citrate), following incubation with alkaline phosphatase conjugated anti-digoxigenin antibody and chromogenic reaction with a nitro blue tetrazolium chloride/5-bromo-4-chloro-3-indolyl phosphate, toluidine salt solution. After staining, they were treated with $10 \% \mathrm{H}_{2} \mathrm{O}_{2}$ in phosphate-buffered saline to bleach the melanophore of the tadpoles, although some of the melanophores remained in the specimens. Following staining, some of the specimens were embedded in $4 \%$ agar in phosphate-buffered saline, and sagittally sliced at $30-\mu \mathrm{m}$ intervals using a vibratome VT1000S (Leica Biosystems, Germany).

Immunohistochemistry. Immunohistochemistry was performed essentially as described previously ${ }^{29}$. In brief, tadpole tails were amputated at $5 \mathrm{dpf}$ (stage 39-41) and fixed at $3 \mathrm{dpa}$ (stage 47) with MEMFA fixative, embedded with $25 \%$ gelatin $15 \%$ sucrose, frozen, sagittally sliced at $10 \mu \mathrm{m}$ thick, and incubated with anti-phospho-Stat3 (Tyr705) mouse antibody (Cell Signalling Technology, MA, \#9138S) following incubation with Alexa Fluor 555-conjugated anti-mouse IgG goat antibody (Invitrogen, A-21424). Nuclei were counterstained with $10 \mu \mathrm{g} / \mathrm{ml}$ of Hoechst 33342 (Lonza, Switzerland).

Knockdown experiment. gRNAs ${ }^{30}$ and cas $9^{31}$ mRNA were designed and synthesised essentially as described previously with minor modifications. In brief, DR2 $74^{30}$ plasmid was digested with Bsa I-HF (New England Biolabs, MA), annealed with oligonucleotide, and they were ligated. Some of the gRNA template plasmids were assembled using a PCR-amplified DR274 fragment, an oligonucleotide, and an In-Fusion HD Cloning Kit (TaKaRa). After transformation and plasmid extraction, the plasmid was digested with Dra I (Aha III; TaKaRa), following PCR amplification and in vitro transcription using AmpliScribe T7-Flash Transcription Kit (Epicentre, WI). The gRNA was purified using RNeasy mini kit. The number of possible off-target sites of the obtained gRNAs was predicted by searching Xenopus laevis genome 9.1 (Xenbase ${ }^{32,33}$ ) using Cas-OFFinder ${ }^{34}$ and possible gRNAs that recognised any sites other than the target genes in the genome were not used. gRNA target sequences were as follows: \#1U.LS; GGTGCATAGCC ATGTTTAGAAGG, \#1D.L; GGACTATGCAACACGTGTTCTGG, \#1D.S; GGACTTTGCAACACGTGTTCTGG, \#2U.L; GCACCATATGCCACGTGTCAC GG, \#2U.S; GTTCCGTGACACGTGGTATATGG, \#2D.L; GAGGTGGAAATGAC GGAAAATGG, \#2D.S; GACGTGGAAATGATGGAAGATGG. For cas 9 mRNA synthesis, pXT7-hcas $9^{31}$ (China Zebrafish Resource Center, China, CZP2) was digested with $\mathrm{Xba}$ I (TaKaRa), following in vitro transcription using mMESSAGE mMACHINE T7 ULTRA Kit (Invitrogen). The obtained cas 9 mRNA was purified using RNeasy mini kit.

The injections were carried out essentially as described previously ${ }^{29}$ with the modifications. In brief, fertilised eggs obtained by artificial fertilisation were 
dejellied by incubation with 3\% cysteine, following injection in Ficoll solution (2\% Ficoll 400 in $10 \mathrm{mM} \mathrm{NaCl}, 0.2 \mathrm{mM} \mathrm{KCl}, 0.1 \mathrm{mM} \mathrm{MgCl}_{2}, 0.2 \mathrm{mM} \mathrm{CaCl}_{2}, 0.5$ mM 2-(4-(2-Hydroxyethyl)-1-piperazinyl)ethanesulfonic acid, $\mathrm{pH}$ 7.5) using Nanoject II (Drummond Scientific Company, PA). We injected cas 9 mRNA (700 $\mathrm{ng} / \mu \mathrm{l})$ and gRNAs $(20 \mathrm{ng} / \mu \mathrm{l}$ each, \#1U.LS, \#1D.L, and \#1D.S in $i l-11 \mathrm{KD} \# 1$, and \#2U.L, \#2U.S, \#2D.L, and \#2D.S in il-11 KD \#2) at a volume of $18.4 \mathrm{nl}$ into single-cell stage embryos. Embryos were injected and kept at $12{ }^{\circ} \mathrm{C}$ until gastrulation began, and reared at $20^{\circ} \mathrm{C}$ thereafter. The tails were amputated from the tadpoles at $6 \mathrm{dpf}$, and the tail regeneration length was measured at $7 \mathrm{dpa}$.

Genomic DNA was extracted using DNeasy Blood and Tissue kit (Qiagen) or by boiling tissues in $50 \mathrm{mM} \mathrm{NaOH}$ at $98^{\circ} \mathrm{C}$ for 10 min followed by adding $1 / 10$ volume of $1 \mathrm{M}$ Tris- $\mathrm{HCl}$ ( $\mathrm{pH}$ 7.5). Primers used for amplification of genomic regions around the gRNA target sites and annealing temperatures were as follows: PIU.L and P1D.L; 5'-TGACTCAGTACTGCGATATTTCCA-3' and 5' ${ }^{\prime}$-AGGTCC TAAAATGTCCCGGT- $3^{\prime}\left(53^{\circ} \mathrm{C}\right)$, P1U.S and P1D.S; $5^{\prime}$-TTTTGCTGGGACTTCG TTGC- $3^{\prime}$ and $5^{\prime}-$ ACCTCAGGCGTTTGATGCTA- $3^{\prime}\left(58^{\circ} \mathrm{C}\right)$, P2U.L and P2D.L $5^{\prime}$-AGAGTTTCTGGATAATGGACCCC- $3^{\prime}$ and $5^{\prime}$-ATATGTGCCCGTTCTGCA GT- $3^{\prime}\left(60^{\circ} \mathrm{C}\right)$, P2U.S and P2D.S; 5'-GGAGAGATAACTAAAAGCTAACACAA- $3^{\prime}$ and $5^{\prime}$-ACATCAAGGATGGAGGTTTGTGA- $3^{\prime}\left(60^{\circ} \mathrm{C}\right)$. Primers used for amplification of positive control efl $\alpha$ were the same as described in the qRT-PCR section. PCR products were cloned into pGEM-T easy vector (Promega, Madison, WI, USA) and sequenced.

The statistical significance of the difference in the relative regeneration length normalised by snout to vent length was assessed by Dunnett's test or Student's $t$-test with a cutoff of $P<0.05$.

Rescue experiment. The rescue construct was assembled using the In-Fusion HD Cloning Kit (TaKaRa). In brief, the il-11 fragment was PCR-amplified using the il-11.L sequence (AB933563) ${ }^{4}$ cloned into pGEM-T easy vector (Promega). Fragments containing acgfp, or minimal CMV promoter and Tet-On Advanced transactivator were PCR-amplified using pAcGFP1-N1 (TaKaRa), or pTet-On Advanced (TaKaRa) as templates, respectively. Oligonucleotides for Gly-Ser-GlyP2A peptide ${ }^{35}$ and the gRNA \#1U.LS target sequence were as follows: $5^{\prime}$-GGAA GCGGAGCTACTAACTTCAGCCTGCTGAAGCAGGCTGGAGACGTGGAGGA GAACCCTGGACCT-3' and 5'-AGGTCCAGGGTTCTCCTCCACGTCTCCAGC CTGCTTCAGCAGGCTGAAGTTAGTAGCTCCGCTTCC-3', 5' -CAGCTCGAC CAAGCTCCTTCTAAACATGGCTATGCACC- $3^{\prime}$ and $5^{\prime}$-AAATCTCGCCAAGC TGGTGCATAGCCATGTTTAGAAGG-3'. These fragments and oligonucleotides were inserted into pTRE-Tight (TaKaRa). To prevent double-strand breaks, synonymous substitutions were introduced at the target sequence for \#1U.LS and \#1D.L in the il-11.L sequence of the rescue construct by PCR using mutated primers as follows: 5' $^{\prime}$ TGATGATTTGCTAAACATGGCTATGCAC- $3^{\prime}$ and 5'- TTTAGCAAATCATCAAATTCCACTTTT-3', 5'-TTGTTCTGATGAAGAAC AAGTTGGGA- $3^{\prime}$ and $5^{\prime}$-TCTTCATCAGAACAAGAACACGTGTT- $3^{\prime}$.

For construction of the plasmid used for the forced expression of $i l-6$, the il-11 sequence of the $i l-11$ rescue construct was substituted by the coding sequence of il-6.S cloned into a pGEM-T easy vector using the In-Fusion system.

Injection was carried out as described in the knockdown experiment section except that $6 \mathrm{ng} / \mu \mathrm{l}$ of the rescue construct was added to the injection solution. Tails from injected tadpoles were amputated at $6 \mathrm{dpf}$, tadpoles were treated with $50 \mu \mathrm{g} /$ $\mathrm{ml}$ doxycycline in $0.2 \%$ salt water as described previously ${ }^{29}$ for 7 days immediately after tail amputation, and tail regeneration length was measured. Only tadpoles showing green fluorescence near the amputation plane or a regenerated tail were used in the acgfp-expressing and $i l-11$ rescue groups. Tadpoles showing extremely high green fluorescence were excluded to prevent possible adverse effects of a high concentration of AcGFP. This criterion was not pre-established.

In experiments comparing the regeneration length of the untreated and doxycycline-treated groups, tadpoles were incubated in either $0.1 \times$ Steinberg's solution (0.3 mM 2-(4-(2- hydroxyethyl)-1-piperazinyl)ethanesulfonic acid, $\mathrm{pH} 7.4$, including $\left.5.8 \mathrm{mM} \mathrm{NaCl}, 67 \mu \mathrm{M} \mathrm{KCl}, 34 \mu \mathrm{M} \mathrm{Ca}\left(\mathrm{NO}_{3}\right)_{2}, 83 \mu \mathrm{M} \mathrm{MgSO}{ }_{4}\right)$ or $0.1 \times$ Steinberg's solution with $50 \mu \mathrm{g} / \mathrm{ml}$ of doxycycline after tail amputation, and the regeneration length of all normally grown tadpoles were measured.

The statistical significance of the difference in the relative regeneration length normalised by snout to vent length was assessed by Tukey-Kramer's test with a cutoff of $P<0.05$

RNA-sequencing. Library construction, sequencing, and mapping were performed essentially as described previously ${ }^{4}$ with the minor modifications. In brief, tail stump tissues cut at the level $0.5 \mathrm{~mm}$ anterior from the amputated plane were collected at 2 dpa from 20 cas 9 mRNA-injected or $i l-11 \mathrm{KD} \# 1$ tadpoles from 3 different batches. Total RNA was extracted using RNeasy mini kit. cDNA library was produced using TruSeq Stranded Total RNA with Ribo-Zero Gold LT Sample Prep Kit (Illumina, CA). We produced a set of approximately $5 \times 10^{7}$ single end reads ( $66 \mathrm{bp}$ ) from each cDNA library using Hiseq 2500 (Illumina). X. laevis genome 9.1 (Xla.v91.repeatMasked.fa; Xenbase ${ }^{32,33}$ ) was used as a reference for mapping. The number of reads mapped to each gene was counted using HTSeq ${ }^{36}$ with a reference gene model (XL_9.1_v1.8.3.2.primaryTranscripts.gff3; Xenbase ${ }^{32,} 33$ ) and compared using DESeq $2^{37}$. A volcano plot was created using ggplot $2^{38}$. Gene expression levels of mouse homologues were obtained from BioGPS $^{39-41}$ using a microarray data set of a previous study in mouse ${ }^{42}$.
Forced expression in intact tail. The gRNA \#tyr target sequence was as follows 5'-GGCTCCATGTCTTCCGTCCAAGG-3'. Although an off-target sequence was found in the latest Xenopus laevis genome 9.1, most tadpoles survived after injection. Construction for forced expression of $i l-11$ was carried out as described in the rescue experiment section except that no mutation was introduced in il-11 sequences, and the gRNA \#tyr target sequence was introduced instead of \#1U.LS using oligonucleotide as follows: ${ }^{\prime}$-CAGCTCGACCAAGCTGGCTCCATGTCTT CCGTCCAAGG- ${ }^{\prime}$ and $5^{\prime}$-AAATCTCGCCAAGCTCCTTGGACGGAAGACATG GAGCC-3'

Injection and doxycycline treatment were carried out as described in the rescue experiment section except that concentration of gRNA \#tyr was $40 \mathrm{ng} / \mu \mathrm{l}$, and embryos were maintained at $20^{\circ} \mathrm{C}$ after they developed to the 16 -cell stage. Doxycycline treatment started at $5 \mathrm{dpf}$, and continued for 2 days following sampling for qRT-PCR or ISH. Tadpoles with GFP signals in the region approximately corresponding to the tail tissues used for qRT-PCR were used for the analyses.

Tissue section ISH. ISH was performed essentially as described previously $y^{43}$. In brief, tadpoles were fixed with Bouin's fixative, embedded in Paraplast (McCormick Scientific, St. Louis, MO). Eight micrometre-thick sagittal sections was prepared, and rehydrated. The slides were incubated with digoxigenin-labelled probe solution overnight, following incubation with alkaline phosphatase conjugated anti-digoxigenin antibody and chromogenic reaction with a nitro blue tetrazolium chloride/5-bromo-4-chloro-3-indolyl phosphate, toluidine salt solution.

For detection of $d c x$, tadpoles were fixed with MEMFA instead of Bouin's fixative. Three probes targeting different regions of $d c x$ were mixed and used to increase detection sensitivity.

Data availability. RNA-sequencing data have been deposited in the DNA DataBank of Japan under accession code PRJDB5211.

We used already deposited GSE10246 data set ${ }^{42}$ in Gene Expression Omnibus of NCBI for expression analysis in mouse tissues.

High resolution supplementary figures are available at doi:10.6084/m9. figshare.5131867.

We declare that all data supporting the findings of this study are available within the article and its supplementary information files or from the corresponding author upon reasonable request.

Received: 1 November 2016 Accepted: 10 July 2017

Published online: 08 September 2017

\section{References}

1. Stoick-Cooper, C. L., Moon, R. T. \& Weidinger, G. Advances in signaling in vertebrate regeneration as a prelude to regenerative medicine. Genes Dev. 21, 1292-1315 (2007).

2. Gargioli, C. \& Slack, J. M. W. Cell lineage tracing during Xenopus tail regeneration. Development 131, 2669-2679 (2004).

3. Kragl, M. et al. Cells keep a memory of their tissue origin during axolotl limb regeneration. Nature 460, 60-65 (2009).

4. Tsujioka, H., Kunieda, T., Katou, Y., Shirahige, K. \& Kubo, T. Unique gene expression profile of the proliferating xenopus tadpole tail blastema cells deciphered by RNA-sequencing analysis. PLoS ONE 10, e0111655 (2015).

5. Ernst, M. \& Putoczki, T. L. Molecular pathways: IL11 as a tumor-promoting cytokine-translational implications for cancers. Clin. Cancer Res. 20, 5579-5589 (2014).

6. Karow, J. et al. Mediation of interleukin-11-dependent biological responses by a soluble form of the interleukin-11 receptor. Biochem. J. 318, 489-495 (1996).

7. Matadeen, R., Hon, W. C., Heath, J. K., Jones, E. Y. \& Fuller, S. The dynamics of signal triggering in a gp130-receptor complex. Structure 15, 441-448 (2007).

8. Yin, T. et al. Involvement of IL-6 signal transducer gp130 in IL-11-mediated signal transduction. J. Immunol. 151, 2555-2561 (1993).

9. Stahl, N. et al. Choice of STATs and other substrates specified by modular tyrosine-based motifs in cytokine receptors. Science 267, 1349-1353 (1995).

10. Zhong, Z., Wen, Z. \& Darnell, J. E. Jr. Stat3: a STAT family member activated by tyrosine phosphorylation in response to epidermal growth factor and interleukin-6. Science 264, 95-98 (1994).

11. Akira, S. et al. Molecular cloning of APRF, a novel IFN-stimulated gene factor 3 p91-related transcription factor involved in the gp130-mediated signaling pathway. Cell 77, 63-71 (1994).

12. Fukada, T. et al. Two signals are necessary for cell proliferation induced by a cytokine receptor gp130: involvement of STAT3 in anti-apoptosis. Immunity $\mathbf{5}$, 449-460 (1996). 
13. Fuhrer, D. K. \& Yang, Y. C. Activation of Src-family protein tyrosine kinases and phosphatidylinositol 3-kinase in 3T3-L1 mouse preadipocytes by interleukin-11. Exp. Hematol. 24, 195-203 (1996).

14. Hirano, T. et al. Complementary DNA for a novel human interleukin (BSF-2) that induces B lymphocytes to produce immunoglobulin. Nature 324, 73-76 (1986).

15. Smith, A. G. et al. Inhibition of pluripotential embryonic stem cell differentiation by purified polypeptides. Nature 336, 688-690 (1988).

16. Williams, R. L. et al. Myeloid leukaemia inhibitory factor maintains the developmental potential of embryonic stem cells. Nature 336, 684-687 (1988).

17. Peterson, H. et al. Qualitative modeling identifies IL-11 as a novel regulator in maintaining self-renewal in human pluripotent stem cells. Front. Physiol. 4, 303 (2013).

18. Fang, Y. et al. Translational profiling of cardiomyocytes identifies an early Jak1/Stat3 injury response required for zebrafish heart regeneration. Proc. Natl Acad. Sci. USA 110, 13416-13421 (2013).

19. Auer, T. O., Duroure, K., De Cian, A., Concordet, J.-P. \& Del Bene, F. Highly efficient CRISPR/Cas9-mediated knock-in in zebrafish by homologyindependent DNA repair. Genome Res. 24, 142-153 (2014).

20. Von Dassow, G., Schmidt, J. E. \& Kimelman, D. Induction of the Xenopus organizer: expression and regulation of Xnot, a novel FGF and activin-regulated homeo box gene. Genes Dev. 7, 355-366 (1993).

21. Gont, L. K., Steinbeisser, H., Blumberg, B. \& de Robertis, E. M. Tail formation as a continuation of gastrulation: the multiple cell populations of the Xenopus tailbud derive from the late blastopore lip. Development 119, 991-1004 (1993).

22. Pallafacchina, G. et al. An adult tissue-specific stem cell in its niche: a gene profiling analysis of in vivo quiescent and activated muscle satellite cells. Stem Cell Res. 4, 77-91 (2010).

23. Ogawa, R. et al. Doublecortin marks a new population of transiently amplifying muscle progenitor cells and is required for myofiber maturation during skeletal muscle regeneration. Development 142, 51-61 (2015).

24. Park, B. Y., Hong, C. S., Weaver, J. R., Rosocha, E. M. \& Saint-Jeannet, J. P. Xaml1/Runx1 is required for the specification of Rohon-Beard sensory neurons in Xenopus. Dev. Biol. 362, 65-75 (2012).

25. Des Portes, V. et al. A novel CNS gene required for neuronal migration and involved in X-linked subcortical laminar heterotopia and lissencephaly syndrome. Cell 92, 51-61 (1998).

26. Tober, J., Maijenburg, M. W. \& Speck, N. A. Taking the Leap: Runx1 in the Formation of Blood from Endothelium. Curr. Top. Dev. Biol. 118, 113-162 (2016).

27. Kang, J. et al. Modulation of tissue repair by regeneration enhancer elements. Nature 532, 201-206 (2016)

28. Nieuwkoop, P. D. \& Faber, J. Normal table of Xenopus laevis (Daudin). A Systematical and Chronological Survey of the Development from the Fertilized Egg till the end of Metamorphosis (Garland Science, 1994).

29. Hoppler, S. \& Vize, P. D. Xenopus Protocols: Post-Genomic Approaches (Humana Press, 2012).

30. Hwang, W. Y. et al. Efficient genome editing in zebrafish using a CRISPR-Cas system. Nat. Biotechnol. 31, 227-229 (2013).

31. Chang, N. et al. Genome editing with RNA-guided Cas9 nuclease in zebrafish embryos. Cell Res. 23, 465-472 (2013).

32. James-Zorn, C. et al. Xenbase: expansion and updates of the Xenopus model organism database. Nucleic Acids Res. 41, D865-D870 (2013).

33. Session, A. M. et al. Genome evolution in the allotetraploid frog Xenopus laevis. Nature 538, 336-343 (2016).

34. Bae, S., Park, J. \& Kim, J. S. Cas-OFFinder: a fast and versatile algorithm that searches for potential off-target sites of Cas9 RNA-guided endonucleases. Bioinformatics 30, 1473-1475 (2014).

35. Kim, J. H. et al. High cleavage efficiency of a $2 \mathrm{~A}$ peptide derived from porcine teschovirus-1 in human cell lines, zebrafish and mice. PLoS ONE 6, e18556 (2011).

36. Anders, S., Pyl, P. T. \& Huber, W. HTSeq-A Python framework to work with high-throughput sequencing data. Bioinformatics 31, 166-169 (2015).
37. Love, M. I., Huber, W. \& Anders, S. Moderated estimation of fold change and dispersion for RNA-seq data with DESeq2. Genome Biol. 15, 550 (2014).

38. Wickham, H. ggplot2: Elegant Graphics for Data Analysis (Springer-Verlag New York, 2009).

39. Wu, C. et al. BioGPS: an extensible and customizable portal for querying and organizing gene annotation resources. Genome Biol. 10, R130 (2009).

40. Wu, C., MacLeod, I. \& Su, A. I. BioGPS and MyGene.info: organizing online, gene-centric information. Nucleic Acids Res. 41, D561-D565 (2013).

41. Wu, C., Jin, X., Tsueng, G., Afrasiabi, C. \& Su, A. I. BioGPS: building your own mash-up of gene annotations and expression profiles. Nucleic Acids Res. 44, D313-D316 (2016)

42. Lattin, J. E. et al. Expression analysis of G protein-coupled receptors in mouse macrophages. Immunome. Res. 4, 5 (2008).

43. Fukazawa, T., Naora, Y., Kunieda, T. \& Kubo, T. Suppression of the immune response potentiates tadpole tail regeneration during the refractory period. Development 136, 2323-2327 (2009).

\section{Acknowledgements}

This study was partly supported by Grant-in-Aids for JSPS Research Fellow from Japan Society for the Promotion of Science (JSPS) (JSPS KAKENHI Grant Number JP15J10835), Challenging Exploratory Research from JSPS (JSPS KAKENHI Grant Numbers JP26640051 and JP16K14590), Scientific Research on Innovative Areas 'Homeostatic Inflammation' (JSPS KAKENHI Grant Number JP24117705) from ISPS, Scientific Research on Innovative Areas 'CHROMOSOME ORCHESTRATION SYSTEM' (JSPS KAKENHI Grant Number JP15H05976) from JSPS, Grant-in-Aid for Scientific Research (A) (JSPS KAKENHI Grant Number JP15H02369) from ISPS, and the Platform Project for Supporting in Drug Discovery and Life Science Research (Platform for Drug Discovery, Informatics, and Structural Life Science) from Japan Agency for Medical Research and Development (AMED).

\section{Author contributions}

H.T. designed and performed most experiments. Y.K. made library and sequenced for RNA-sequencing experiment. T.F., T.Kunieda, K.S. and T.Kubo wrote manuscript.

\section{Additional information}

Supplementary Information accompanies this paper at doi:10.1038/s41467-017-00594-5.

Competing interests: The authors declare no competing financial interests.

Reprints and permission information is available online at http://npg.nature.com/ reprintsandpermissions/

Publisher's note: Springer Nature remains neutral with regard to jurisdictional claims in published maps and institutional affiliations.

Open Access This article is licensed under a Creative Commons Attribution 4.0 International License, which permits use, sharing, adaptation, distribution and reproduction in any medium or format, as long as you give appropriate credit to the original author(s) and the source, provide a link to the Creative Commons license, and indicate if changes were made. The images or other third party material in this article are included in the article's Creative Commons license, unless indicated otherwise in a credit line to the material. If material is not included in the article's Creative Commons license and your intended use is not permitted by statutory regulation or exceeds the permitted use, you will need to obtain permission directly from the copyright holder. To view a copy of this license, visit http://creativecommons.org/ licenses/by/4.0/

(C) The Author(s) 2017 